\title{
Communauté culturelle de lecteurs et d'auteurs en interaction : Construction langagière et identitaire à travers une correspondance scolaire
}

\author{
Marlène Lebrun \\ Université de Provence - I.U.F.M. Aix-Marseille \\ CIELAM Aix-Marseille \\ c.romain@aix-mrs.iufm.fr \\ Christina Romain \\ Université de Provence - I.U.F.M. Aix-Marseille \\ Laboratoire Parole et Langage - U.M.R. 6057 - C.N.R.S. \\ marlene.lebrun@hotmail.com
}

\begin{abstract}
L'objectif de la recherche descriptive, dont nous nous proposons de présenter les principaux résultats à l'occasion de cet article, est de montrer comment une enseignante développe dans sa classe une communauté culturelle de lecteurs et d'auteurs en interaction. Cette recherche à dimension internationale a été conduite dans une classe française de cours préparatoire ${ }^{1}$. Le corpus a été constitué par des données recueillies sur une période de 7 mois (septembre à mars d'une même année scolaire). A raison d'une journée par semaine, cette classe de $\mathrm{CP}$ a constitué un terrain d'observation ayant donné lieu à des recueils de données (verbatim de séquences d'apprentissage, enregistrements audio et vidéo, analyse des productions intermédiaires et finales, entretiens de quelques élèves représentatifs de la diversité socioculturelle de la classe). Plus précisément, l'objectif est de décrire comment, à travers un projet reposant sur un échange culturel ${ }^{2}$, le rapport à la culture des élèves va évoluer grâce aux échanges liés à un projet international et fédérateur reposant sur la prise de conscience de sa propre culture mais aussi de celle de l'autre, et comment ce type de projet, reposant sur un registre discursif très interactif de l'enseignante, peut avoir des incidences sur certaines performances linguistiques et culturelles. Notre recherche a constitué à décrire et à analyser un projet reposant sur un échange culturel ${ }^{3}$. Plus précisément, nous avons souhaité analyser l'impact d'un projet culturel de correspondance internationale (Canada / France) sur les interactions entre la construction langagière et identitaire de jeunes élèves marseillais socio culturellement peu favorisés ${ }^{4}$ et l'ouverture à une culture autre. Alors que nous avons largement publié des analyses de compétences générées par le projet épistolaire ${ }^{5}$, nous nous centrerons sur l'analyse des interactions discours / dispositifs / performances tant au plan linguistique qu'à celui culturel en nous appuyant sur nos références théoriques comme fondement de nos hypothèses de recherche et de notre méthodologie d'observation en milieu écologique, de recueil et traitement des données. Pour conduire cette analyse, nous avons donc retenu trois axes : le positionnement des élèves dans leur propre culture et l'appropriation de culture des autres, l'évolution de leurs représentations et de leur rapport à la culture et l'impact du registre discursif de l'enseignante sur certaines performances linguistiques et culturelles, à travers notamment les interactions entre la construction langagière et identitaire de jeunes élèves marseillais dits socio-culturellement peu favorisés ${ }^{6}$ et l'ouverture à une culture différente.
\end{abstract}

Pour ce faire, nous avons analysé les interactions didactiques et pédagogiques entre l'enseignante et ses élèves dans différentes situations : d'une part les productions narratives orales concernant l'exploitation des ouvrages du comité de lecture ${ }^{7}$ destinés à être partagés avec les correspondants québécois, et d'autre part les données culturelles liées à la construction identitaire et au rapport à l'autre. Dans un premier temps, une «carte descriptive » du fonctionnement des interactions langagières dans la classe a donc été dressée en pointant la position des élèves et de l'enseignante. Cette description visant à identifier et décrire le registre discursif de l'enseignante afin de mieux comprendre les enjeux du projet mis en place sur cette année scolaire. Puis nous avons analysé les données. Un premier corpus oral a été constitué par les enregistrements des élèves de la classe de $\mathrm{CP}$ portant sur des récits narratifs oraux faisant suite à une 
histoire lue par l'enseignante, appartenant à celles évaluées en comité de lecture. Ce corpus a fait l'objet d'une comparaison avec une classe de CP du même établissement ne travaillant pas sur le projet culturel et dont le registre discursif de l'enseignante ne correspondait pas à celui de l'enseignante de la classe étudiée. Nous avons donc demandé à cette enseignante, appartenant au même établissement scolaire, de se livrer au même exercice à la suite duquel nous avons recueilli les corpus oraux des élèves afin de les analyser et de les comparer avec ceux du CP1. Les données ont été transcrites et ont donné lieu à une analyse comparative conduite en fonction des outils linguistiques utilisés : organisateurs textuels et connecteurs, progression thématique, emploi des temps, procédés anaphoriques et structure/organisation narrative. Cette étude a été réalisée sur deux périodes de l'année scolaire : au mois de septembre puis au mois de décembre. Un second corpus a été constitué par un questionnaire passé à deux reprises (en septembre et en mars) et à plusieurs entretiens reposant sur le rapport à la culture et sur le rapport à la littérature entretenus par les élèves et ont été conduits toute l'année par les chercheurs auprès des élèves de la classe de CP1. L'analyse de ce corpus vise à mettre en avant à la fois les représentations et les connaissances des élèves sur leur ville, sur leurs correspondants et sur leur milieu de vie et à se demander comment et selon quelle intensité, ces élèves se positionnent dans leur propre culture et pénètrent la culture des autres.

L'identification et l'analyse des compétences générées par les différentes actions du projet de correspondance culturelle a été au cœur de nos préoccupations et notre analyse s'est donc centrée sur les interactions discours / dispositifs / performances tant au plan linguistique qu'à celui culturel. Reposant sur un registre discursif spécifique, le projet de classe de l'enseignante vise à établir une communauté d'élèves optimisant les échanges et le dialogue entre des élèves marseillais et des élèves québécois et leurs professeurs respectifs. Notre rôle a été d'identifier et d'analyser les compétences générées par les différentes actions reposant sur un registre discursif spécifique qui vise à établir une communauté d'élèves optimisant les performances tant linguistiques que culturelles liées à la construction identitaire Dans la mesure où les compétences se traduisent en performances, nous adopterons la définition de performance donnée par l'équipe THEODILE : « Dans performance, on entend aussi bien les savoirs acquis que les connaissances, les savoir-faire, les rapports à, les attitudes, les conduites, les compétences (ou capacités), bref tous les contenus enseignables et / ou apprenables dont on cherche à analyser la maîtrise chez les élèves » (Daunay, 2007).

\section{Fondements théoriques}

Ils sont au nombre de trois: les positionnements dans l'interaction didactique, les performances linguistiques au service de la structuration du récit narratif oral et le « rapport à » la culture.

\subsection{Les positions dans l'interaction didactique}

A l'origine les participants d'une situation d'enseignement ont des «positions statutaires » (Vion, 1992, 78), asymétriques et définies par l'institution (position d'élève et position de professeur), qui déterminent leurs places respectives dans l'interaction didactique. L'analyse proposée vise dans un premier temps à étudier le registre discursif de l'enseignante afin de décrire la gestion de la relation interpersonnelle enseignante / élève, la façon dont l'enseignante envisage et met en place la relation interpersonnelle mais aussi interdiscursive dans la classe. Notre analyse s'inspire donc de travaux menés tant en pragmatique qu'en linguistique interactionnelle et plus précisément de ceux de Kerbrat-Orecchioni (1992) et de Vion $(1992,1999)$. Le cadre d'analyse a (donc) pour objet principal de décrire les relations qui se construisent, par le biais de l'échange verbal, entre les interactants eux-mêmes, à savoir le professeur et ses élèves, et à les confronter aux représentations du corps enseignant. Le modèle de Brown et Levinson $(1978,1987)$ prolongé par Kerbrat-Orecchioni (1996) ainsi que la notion de face de Goffman $(1971,1974)$ seront tout particulièrement utiles pour mettre en évidence la gestion de cette relation interpersonnelle. La notion de distance interpersonnelle et la notion de politesse, en tant que procédés que le locuteur met en œuvre pour ménager ou valoriser son partenaire d'interaction, seront privilégiées. 
Afin d'étudier la variable de la gestion de la relation interpersonnelle certains indicateurs sont retenus : relation complémentaire, déictiques de personne, impératif, prénom de l'élève, tours de parole (interruption, chevauchement, silence, écoute attentive, etc.), mimogestualité, faits prosodiques et faits discursifs. L'analyse a porté sur les différentes séances composant une journée de cours afin d'étudier comment l'enseignante structure et gère sa classe sur les plans organisationnel et relationnel. L'objectif est de décrire le registre discursif de l'enseignante et de déterminer, dans quelle mesure, ce dernier peut avoir une incidence plus ou moins importante sur les performances linguistiques et culturelles des élèves.

\subsection{La structuration du récit oral}

$\mathrm{Au}$ cycle des apprentissages fondamentaux, les élèves de CP continuent leur apprentissage du langage oral à travers le « respect de l'organisation de la phrase, expression des relations de causalité et des circonstances temporelles et spatiales (pourquoi ? quand ? où ?) ; utilisation plus adéquate de la conjugaison, emploi d'un vocabulaire de plus en plus diversifié ; prises de parole de plus en plus longues et mieux organisées, dans le respect des sujets traités et des règles de la communication » (B.O. H.S. $n^{\circ} 3$, 19 juin 2008, Programmes d'enseignement de l'école primaire, 17). En respectant les recommandations officielles, l'enseignante a institué des comités de lecture à l'occasion desquels ses élèves sont amenés à écouter régulièrement des textes lus par l'enseignante. La compréhension s'évalue à travers la reformulation orale du récit. Les élèves sont invités également à poser des questions et à effectuer des lectures silencieuses.

La restitution orale des récits d'albums lus en lecture offerte, c'est-à-dire dans des espaces temps réservés à la lecture magistrale exclusive de toute autre activité et visant notamment le développement de l'envie de lire mais aussi dans une moindre mesure les échanges, permet d'observer les performances linguistiques en jeu. Les comités de lecture reposent sur des ensembles de genres textuels adaptés à des situations de communication ou d'activités déterminées. Dolz et Pasquier (1993) ainsi que Schneuwly et Dolz (1997) insistent sur l'importance de «rendre l'élève conscient de cette correspondance, et de le rendre apte à choisir les modèles de genres pertinents pour une activité langagière donnée » mais aussi, « sachant que les élèves rencontrent divers problèmes linguistiques lors de la rédaction ou de la lecturecompréhension de tout texte », de leur permettre d'accéder à la « maitrise technique de sous-ensembles de processus de structuration des textes ». Dans cette perspective, Dolz, Noverraz et Schneuwly (2001) soulignent la nécessaire prise en considération par les enseignants, dans la construction de leurs séquences, de l'utilité, de la finalité et de la fonctionnalité «scolaire» du genre à enseigner, de la constitution d'un corpus d'exemplaires de textes relevant du genre considéré et en adéquation aux capacités des élèves et, bien sûr, de la prise en compte du genre ainsi que des «pratiques sociales de référence » associées. Dans le cadre du travail mené par l'enseignante à travers le comité de lecture, nous nous sommes intéressées au recours aux différents indicateurs textuels mis au service de la cohérence du récit : étapes de la structure narrative, connecteurs, procédés anaphoriques, progression thématique et emploi des temps. Charolles (1978) souligne ainsi l'importance des conditions à réunir pour qu'un texte puisse être considéré comme bien formé. Il insiste sur les règles permettant d'assurer la cohérence d'un texte : références, progression, non contradiction et relation. Notre étude de la structure textuelle des récits revêt donc des caractéristiques spécifiques en ce qu'elle permet de mettre en lumière l'acquisition des micro-structures (assurant la cohérence de phrase en phrase) et des macro-structures (assurant la cohérence du texte dans son entier) (Charolles, 1976).

Les notions d'organisateurs textuels, d'emploi des temps, de procédés anaphoriques, de progression thématique et de structure narrative méritent de faire l'objet d'une étude commune car ils participent également à la cohésion du texte narratif. Leur étude conjointe s'inscrit complètement dans une même perspective de recherche.

En effet, un récit narratif n'est pas une suite aléatoire de phrases mais ces différents éléments contribuent et bien souvent interagissent les uns avec les autres. En effet, en français, il y a des temps qui sont propres à la narration, l'imparfait et le passé simple, correspondant pour le premier au cadre de l'action et pour le second à l'action proprement dite; il y a les organisateurs textuels, qui sont là pour participer à la 
cohésion du texte, de tout texte d'ailleurs; il y a également les procédés anaphoriques qui interviennent lorsqu'un mot ou un groupe de mots renvoie à un autre mot ou groupe de mots apparu précédemment dans le discours; il y a encore la progression thématique qui participe à l'apparition et à l'organisation du connu et du nouveau dans un texte; et enfin il y a la structure narrative elle-même qui correspond à une succession de séquences organisées et cohérentes. Rappelons que, selon Weinrich (1973), Benveniste mais aussi Bronckart et al. (1985), les unités spécifiques de la narration sont le passé simple, l'imparfait, les organisateurs temporels et l'absence de futur (à l'exception du futur d'anticipation). Cependant d'après ces auteurs, la singularité d'un texte narratif est assurée avant tout par le sous-système temporel passé avec l'alternance simple / imparfait.

Les connecteurs attireront notamment notre attention puisque selon Fayol, Gombert et Mouchon (1991), la présence ou l'absence des connecteurs, à l'oral aussi bien qu'à l'écrit, tient au fait que la production orale ou écrite doit être structurée et pour ce faire elle doit correspondre à une structure complexe contenant « des obstacles ou des complications ». Ces auteurs ajoutent: « en revanche, tant que les faits rapportés demeurent frustes et/ou banals, seul et est employé » (1991, p.95). Leurs résultats ont confirmé les études relatives à la genèse du récit (Appelbee, 1978 ; Fayol, 1981; Miller et Sperry, 1988 ; UmikerSebeok, 1979 ; Waters et Hou, 1987). Il sera donc significatif de comparer les résultats de notre étude aux résultats convergents de ces différentes études.

Enfin, la progression thématique et les procédés anaphoriques seront particulièrement intéressants à étudier puisque la maîtrise de la progression thématique doit progressivement évoluer tout au long de l'école primaire (d'une progression constante chez l'enfant de 6-7 ans à une progression plus complexe constante et linéaire- chez l'enfant de 10 ans) tout comme la maitrise des les différents procédés anaphoriques (de substitutions pronominales chez l'enfant de 6-7 ans à des procédés diversifiés chez l'enfant de 10 ans -notamment par l'introduction de recours à des substitutions lexicales et relation anaphoriques marquées par les déterminants-) au fil des mois.

A ce stade, nous signalerons enfin une étude menée par Prêteur et Rouquette (1992). Ils ont cherché à appréhender l'influence d'une pédagogie fonctionnelle autour du livre de jeunesse auprès d'enfants d'âge préscolaire, issus de milieux sociaux défavorisés fréquemment touchés par les échecs scolaires. Ils concluent leur étude en soulignant que si cette démarche pédagogique est pertinente quelque soit l'appartenance socioculturelle des élèves, elle accentue néanmoins les écarts entre milieux sociaux contrastés. Si notre propre étude n'est pas une étude contrastive, elle prend néanmoins en compte l'appartenance socioculturelle dite « défavorisée » des élèves.

Ainsi, l'analyse des performances linguistiques sera conduite en comparant deux classes de CP d'un même établissement inscrit en zone d'éducation prioritaire. Il sera intéressant, compte tenu du fait que nous mettrons en relation le registre discursif de l'enseignante et son travail conduit autour de l'album de littérature de jeunesse, de s'interroger sur les relations éventuelles entre le registre discursif de l'enseignante et les performances linguistiques des élèves reposant sur l'exploitation de ce même support.

\subsection{Le rapport à la culture}

Le concept de « rapport à » qui vient de la psychanalyse et sociologie s'est imposé dans le champ de la didactique du français quand elle s'est intéressée au sujet. En s'appuyant sur la définition proposée par Delcambre et Reuter $(2002,19)^{8}$, on retient que plusieurs indicateurs, dont les discours sur et les représentations, permettent d'analyser les images du sujet lecteur auteur de textes littéraires (auxquels ce dernier est le plus souvent confronté sans les avoir choisis) et de tisser son "rapport à » la littérature scolaire et extrascolaire. Le rapport à est donc le fait d'un sujet : en ce sens, il est personnel, distancié et positif à partir du moment où il devient conscient et se verbalise. Il peut même faire l'objet d'un enseignement-apprentissage scolaire. Le professeur gagnera à comprendre les rapports aux objets enseignés de ses élèves et ceux-ci donneront plus de sens aux pratiques disciplinaires en prenant conscience de leur rapport à. Dans l'entrée Rapport à du Dictionnaire des concepts fondamentaux des didactiques, Daunay pointe d'ailleurs certaines difficultés d'apprentissage car le «rapport à » de l'apprenant aux contenus enseignés est primordial dans l' apprentissage : "Le concept de rapport à en 
didactiques désigne la relation (cognitive mais aussi socio-psycho-affective) qu'entretient l'apprenant aux contenus et qui conditionne en partie l'apprentissage de ces derniers : un rapport aux contenus qui ne correspond pas à celui que l'école envisage peut rendre difficile l'accès aux contenus enseignés 》 (Reuter (Ed.), 2007, 191). Finalement, Daunay met en garde contre une réification de la notion de rapport à qui se transforme en équivalent d'handicap socioculturel si on reporte les difficultés de l'élève sur lui seul sans tenir compte du contexte scolaire et social dans lequel il évolue. (Reuter, (Ed.), 2007, 195). Il ajoute : " Le rapport à, dans une telle approche, ne concerne pas en effet le seul élève et ses aptitudes propres, mais aussi l'enseignant et les autres acteurs du système scolaire. C'est encore à la condition de ne pas oublier ce principe que le rapport à peut devenir un contenu d'enseignement.» (Ibid., 196). Par conséquent, il importe d'analyser l'évolution de ce «rapport à » à travers son évolution et son appréhension de la culture « propre » et « autre » dans un projet de correspondance internationale.

Nous allons donc commencer par présenter le registre discursif de l'enseignante qui constitue le cadre explicatif des performances observées.

\section{Le registre discursif de l'enseignante : vers la création d'une communauté d'échanges et de dialogue}

Une «carte descriptive » du fonctionnement des interactions langagières dans la classe est dressée en pointant la position des élèves et de l'enseignante. Cette description vise à identifier et à décrire le registre discursif de l'enseignante afin de mieux comprendre les enjeux du projet. Les principaux traits du registre discursif de l'enseignante sont mis en regard de sa volonté de créer au sein de sa classe une communauté de parleurs, de scripteurs et de lecteurs.

Avant de proposer la description et l'analyse du registre discursif de l'enseignante, nous allons décrire rapidement la classe de CP sujet de notre étude ainsi que les objectifs décrits par l'enseignante en début d'année.

Il s'agit d'une classe de 23 élèves dont 10 élèves sont en très grande difficulté. Certains présentent un retard scolaire jusqu'à 3 ans d'âge et les trois quarts de la classe ont des difficultés diverses concernant le langage oral (difficultés linguistiques (compétences lexicales, syntaxiques et phonologiques) et / ou langagières (compétences de communication et compétences liées au langage d'évocation)) et la compréhension (compréhension orale et compréhension écrite). Par ailleurs cette classe est catégorisée comme étant difficile du fait de problèmes d'absentéisme, de racisme et de violence ainsi que du fait, chez les élèves, de l'absence d'intérêt pour l'objet livre et d'absence de volonté de mise au travail. L'évaluation diagnostique établie à l'occasion des quinze premiers jours de classe met en avant les points suivants :

- Certains élèves entrent au CP sans avoir bénéficié d'un cursus scolaire régulier en maternelle (cf. : registre d'appel de la classe de GS) ; certains d'entre eux étaient même déscolarisés (4\%).

- Sur 22 élèves, un quart soit 6 élèves ont acquis les compétences de fin de cycle 1 (cf. : évaluation nationale début de $\mathrm{CP}$ ) et peuvent suivre un $\mathrm{CP}$ normalement. Il reste 17 élèves qui ont besoin d'un enseignement individualisé pour rattraper le retard scolaire.

- Tous les élèves présentent des difficultés liées à la pratique et à l'expression du langage oral. Certains refusent de parler (timidité, problèmes familiaux, autres), d'autres connaissent des troubles du langage et six sont issus de milieux allophones (CLIN).

- Il n'existe pas ou peu d'interactions entre les élèves: ils ne savent pas travailler en équipe, ils s'approprient le matériel, ils se disputent pendant la répartition des rôles, pas de solidarité entre eux, l'ambiance est tendue et la communication entre pairs difficile.

- Certains élèves étrangers (sans nationalité française à ce moment de l'année scolaire) sont rejetés par reste de la classe. Des problèmes de violence et de discrimination ethnique sont prégnants.

- Les élèves font preuve d'une grande méfiance des parents vis-à-vis de l'école. 
Cette évaluation de début d'année a conduit l'enseignante à établir une série d'objectifs pour l'année scolaire considérée. Parmi lesquels, l'objectif prioritaire est défini par l'enseignante comme celui de « supprimer tous ces problèmes en mettant en œuvre des dispositifs et des actions qui puissent rendre tout le monde heureux ». L'enseignante décide de travailler pendant les deux premières périodes les compétences suivantes : le langage oral (parler sur des images, raconter des histoires et dire des textes), les sonorités de la langue, l'identification des composantes sonores du langage, la compréhension, le dire / redire / raconter et l'écrire, les activités en graphisme et en écriture, le principe alphabétique, le liens entre lecture et écriture, la production d'écrit en dictée à l'adulte (dicter un texte cohérent en adéquation avec le sujet, rester dans le sujet, respecter les consignes, faire preuve d'imagination). Par ailleurs, l'enseignante déclare avoir construit son enseignement autour d'une pédagogie centrée sur l'enfant, ses intérêts, ses besoins et ses aptitudes. Pour ce faire, elle a formé trois groupes de niveau : les forts, les moyens, les faibles. Elle insiste sur le fait que ces trois groupes ne sont pas constitués définitivement : à la fin de chaque période, on procède aux «passages » des groupes inférieurs aux groupes supérieurs (on accède toujours de l'un à l'autre dans l'ordre ascendant; on peut être maintenu dans son groupe; on ne rétrograde pas). Son dispositif, organisé autour de groupes d'élèves, s'articule autour de trois types différents de séance alternant régulièrement :

- des séances fondées sur le système du travail collectif: la leçon traditionnelle et le comité de lecture.

- des séances fondées sur le système du travail par équipe : dans cette configuration, la constitution de groupe homogène sera privilégiée : la lecture par groupe de niveau.

- des séances fondées sur le système du travail individualisé : la lecture libre à haute voix donnant lieu à une présentation du livre choisi à la classe?

L'ambition de l'enseignante s'illustre au travers de deux procédés de travail mis en place sur l'année : le comité de lecture à travers l'élection du livre de la semaine ${ }^{10}$ parmi des livres mis en réseau pour construire un socle de culture commune et la correspondance scolaire avec une classe québécoise.

L'objectif de cette recherche descriptive, dont nous allons présenter les principaux résultats, est donc de montrer comment l'enseignante tente de développer dans cette classe, qui présente des difficultés dès le début de l'année, une communauté culturelle de lecteurs et d'auteurs en interaction.

L'analyse s'appuie sur des extraits d'un enregistrement vidéo pris lors d'une matinée traditionnelle de cours en fin de période 1 (septembre et octobre). A ce stade, et après deux mois de classe, le rapport au travail et à l'autre a évolué très positivement chez les élèves qui sont capables de s'écouter et de travailler ensemble. Ils commencent à s'investir dans le projet culturel et la correspondance franco-québécoise. Néanmoins, l'enseignante souligne que les rythmes d'apprentissage et les niveaux de maîtrise des compétences liées à l'acculturation écrite sont encore extrêmement différenciés à cette période de l'année.

Pendant la période 1 , mais aussi tout au long de l'année, l'enseignante cherche à structurer les échanges entre élèves, à favoriser la parole et l'écoute, à faire respecter les tours de parole, à assurer un lien entre l'écoute, les questions et les réponses. Ainsi, d'emblée, elle ambitionne de réunir et d'encourager la formation d'une communauté de parleurs dans sa classe. Cet objectif vise la réunion (communion), au sens propre et au sens figuré, de ses élèves qui sont issus de cultures différentes (certains élèves / groupes d'élèves sont susceptibles de causer des tensions au sein de la classe). Pour cela, et selon ses propos, elle souhaite mettre au premier plan la «parole» et non pas le «corps» des élèves composant sa classe. Par « corps » l'enseignante entend les caractéristiques culturelles représentant l'image de l'enfant dans sa position extrascolaire (origines familiales, sociales, économiques, etc.) : les enfants qui se connaissant souvent depuis la maternelle se sont regroupés par affinités communautaires et, depuis le début de l'année, les conflits sont nombreux entre eux au sein et hors de l'espace de la classe. L'enseignante développe des procédés pédagogiques et didactiques visant à provoquer la parole et l'écoute des élèves par les élèves (la parole de l'enfant dans la classe) et non pas le corps et ses représentations (donc l'image d'un enfant dans sa position extrascolaire) et dans le même temps elle fait en sorte de s'effacer pour laisser la première place interactive aux élèves. 
Cette volonté pédagogique de l'enseignante s'inscrit dans la lignée des pédagogies alternatives, où l'on trouve la pédagogie coopérative de type Freinet dont l'enseignante dit s'inspirer. Cette pédagogie se caractérise par la place et le rôle accordé aux règles liées à la circulation de la parole en classe. Le rôle du maître étant de faire en sorte que les élèves s'approprient les règles d'écoute et de communication tout en les comprenant et les contractualisant (Reuter, 2007).

L'analyse concrète des procédés discursifs récurrents de l'enseignante, qui structurent son propos et qui visent à valoriser, développer et entretenir non seulement la relation interpersonnelle mais aussi la relation inter-discursive, a été menée dans le cadre de deux activités dont nous allons rapidement présenter des extraits : le regroupement du matin autour de «la parole des élèves » et le déroulement d'une activité conduite en ateliers.

\subsection{Le regroupement du matin autour de « la parole des élèves »}

Deux fois par semaine l'enseignante propose un entretien ritualisé qui est très attendu des élèves. En voici quelques extraits analysés :

« on va commencer la parole » : l'objectif est clairement affiché, rappelé, il s'agit de mettre au centre de l'activité «la parole». Afin de développer cette communauté de parleurs, l'enseignante rappelle régulièrement les règles : «Qui veut s'inscrire pour dire quelque chose, pour la parole? ». Les élèves souhaitant prendre la parole lèvent le doigt et s'inscrivent, l'enseignante leur distribue ensuite la parole à tour de rôle; lorsque l'élève s'arrête de parler, l'enseignante lui demande toujours s'il veut ajouter quelque chose ; un "très bien" de l'enseignante clôture chaque prise de parole par un élève. L'enseignante veille à ce que tous les élèves écoutent la parole de l'enfant inscrit et ne l'interrompent pas. Puis, l'enseignante laisse la parole aux élèves qui ont des questions à poser : «maintenant on va procéder à l'inscription des questions, qui veut poser des questions? », l'objectif étant que les enfants ayant des questions puissent les poser à ceux qui ont demandé la parole dans la première partie de l'activité. L'enseignante surveille la formulation syntaxique de l'adresse des questions et insiste sur la destination: « je voudrais poser une question à Louis ». Elle insiste également sur l'intelligibilité des échanges: «plus fort vos échanges les enfants ». L'enseignante, à nouveau, s'assure du respect et de la gestion des tours de parole et bien sûr de l'écoute (silencieuse et bienveillante) des autres élèves. Lorsqu'une élève interrompt l'enseignante, cette dernière souligne «est-ce que je suis en difficulté ? » et insiste donc, non seulement sur les règles implicites des tours de parole, mais aussi sur l'intérêt de la prise de parole. Elle rappelle l'intérêt d'entrer dans l'interaction en y prenant la parole mais aussi en s'écoutant, il est tout aussi important d'attendre son tour pour prendre la parole «tu attends ton tour», que de bien structurer sa propre intervention « il faut faire une phrase ». Enfin, l'enseignante fait un bilan de l'activité de parole et félicite les élèves qui ont presque tous pris la parole. On constate que des élèves dont les difficultés peu ou prou importantes ont été identifiées se retrouvent parmi les élèves qui ont sollicité spontanément la parole.

\subsection{Le déroulement d'une activité conduite en ateliers}

A l'occasion du travail en petits groupes, l'enseignante s'assure de la répartition des rôles de scripteur et de porte parole. La feuille d'activité est donnée lorsque le choix a été fait. Au bout de plusieurs minutes, elle prend la parole en veillant à expliciter systématiquement son action « la maîtresse a quelque chose à dire »: l'enseignante insiste sur sa prise de la parole et demande donc l'attention de ses élèves. L'enseignante aide alors un groupe puis présente au reste de la classe sa fiche de travail afin de la soumettre aux commentaires. Ce sont donc les élèves qui vont valider ce travail intermédiaire. Les règles de l'activité sont reformulées par les élèves eux-mêmes. Puis, l'enseignante tourne dans les ateliers et s'arrête pour faire travailler le groupe le plus en difficulté. A l'issue de l'activité, chaque groupe présente aux autres groupes son travail qui sera validé tout ou partie et corrigé si besoin (soit directement par l'élève porte-parole qui est venu présenter le travail au tableau soit par un autre élève du groupe soit par un élève appartenant à un autre groupe). 
On remarque que l'enseignante pose un cadre de travail très strict reposant sur des règles précises et une structuration particulière des activités à l'occasion desquelles ce sont les élèves qui (re)formulent les règles et valident le travail des différents groupes. Elle insiste sur la bonne " posture d'écoute » et sur les règles régissant la prise et les tours de parole. L'enseignante distingue clairement le "vous» correspondant aux élèves composant la classe dans son entier (et ce, même, dans la grande majorité des situations de réprimandes individuelles qui sont l'occasion de rappeler les règles de savoir-vivre) $\mathrm{du}$ 《 je » qu'elle associe à « la maîtresse, elle ... ». Elle vise donc à faire prendre conscience à ses élèves qu'ils appartiennent à un groupe dont elle ne fait pas partie mais dont elle est le guide... Elle distingue donc deux plans énonciatifs avec le « moi », « je », « la maîtresse a dit que... » du « vous » désignant la classe, «votre travail» désignant celui de la classe et qui est constitué par l'ensemble des productions individuelles ou de groupes. Elle peut utiliser le « votre groupe, vos avancées » alors qu'elle s'adresse à un seul élève du groupe.

L'enseignante a un rôle de guide et de régulateur et n'hésite pas à se mettre en retrait afin de donner la parole aux élèves. Elle pose le cadre de l'activité puis intervient pour étayer, voire réguler. Le registre discursif de l'enseignante correspond à un registre extra-traditionnel puisqu'elle n'oppose pas sa position haute (dirige, organise, structure, conduit, sanctionne, valide, interrompt, etc.) à la position basse (exécutrice) des élèves mais qu'elle cherche à positionner tous les élèves en position haute, c'est-à-dire dans une position commune, sans discrimination quant aux productions individuelles, où chacun, tour à tour, évalue, motive, discute, valide la production de chacun. Elle donne la parole aux élèves pour reformuler les consignes. Sa position n'est pas haute, au sens traditionnel, mais correspond à une position de guide régulateur d'activités visant à ce que les élèves s'autonomisent au maximum de leurs possibilités respectives. Ils parviennent objectivement, selon des critères identifiés et acceptés par chacun, à diriger, organiser, structurer, conduire, sanctionner et valider leurs propres productions et celles des pairs. Le registre discursif de l'enseignante se trouve donc être en adéquation avec la pédagogie Freinet dont elle s'inspire.

Sur la base de l'analyse de ce registre discursif très interactif, promouvant la parole des élèves et servant de base à la structuration de la relation pédagogique et didactique mais aussi et surtout redistribuant le schéma classique du positionnement des places au sein de l'interaction didactique, nous avons étudié les performances linguistiques à travers la production d'un récit narratif oral et les performances culturelles dans le cadre du projet de correspondance scolaire.

\section{Quelle évolution des performances linguistiques et culturelles ?}

On ne peut dissocier l'apprentissage des contenus comme savoirs et savoir-faire et le rapport à ces contenus construit par l'apprenant. Ainsi en devenant un contenu d'enseignement au même titre qu'un comportement, une attitude, un savoir-être, le rapport à peut s'analyser aussi comme une performance découlant de la situation d'enseignement-apprentissage. Si nous nous appuyons sur la définition proposée par Bertrand Daunay (2007) qui lie les performances aux apprentissages, nous considérons que les performances sont un ensemble de compétences et d'appétences dont l'analyse est liée à la construction du dispositif didactique. Celui-ci permet de mesurer ses effets en compétences (savoirs et savoir-faire spécifiques) et en appétences (représentations, « rapport à », posture).

\subsection{Performances linguistiques}

Pour analyser les performances linguistiques, nous avons choisi de prendre des indicateurs linguistiques qui permettent d'analyser les récits oraux proposés par des élèves de CP à la suite d'une lecture offerte (à haute voix) du professeur. Des enregistrements ont été réalisés et constituent un corpus de récits oraux produits dans le cadre des comités de lecture. Comme nous l'avons déjà évoqué, le corpus a fait l'objet d'une comparaison avec une classe de $\mathrm{CP}$ du même établissement (que nous nommerons CP2 par opposition à CP1). Le CP2 ne travaille pas sur le projet culturel et le registre discursif de l'enseignante est moins interactif que celui de l'enseignante de la classe étudiée. Nous avons donc demandé au 
professeur du CP2 de se livrer au même exercice à la suite duquel nous avons recueilli les corpus oraux des élèves afin de les analyser et de les comparer avec ceux du CP1.

Les données ont été transcrites ${ }^{11}$ pour mener une analyse comparative sur deux périodes de l'année scolaire, au mois de septembre puis au mois de décembre. Le professeur a lu deux histoires différentes, Le petit chaperon rouge ${ }^{12}$ en période 1 et Loulou $^{13}$ de Solotareff en période 2.

Les outils linguistiques suivants ont été retenus: étapes de la structure/organisation narrative, organisateurs textuels et connecteurs, procédés anaphoriques, progression thématique et emploi des temps. L'intérêt(s) de l'étude est de s'interroger sur l'évolution des performances des élèves eu égard aux indicateurs choisis pour établir des liens éventuels entre les performances et la méthodologie pédagogigo-didactique de l'enseignante (CP1). La comparaison avec une classe témoin non impliquée dans le projet culturel est aussi un indicateur utile quand on essaie de mesurer l'impact du registre discursif de l'enseignante et son dispositif didactique.

Suite à l'analyse détaillée des résultats, il apparaît :

- un renforcement de la maîtrise de la structure narrative dans les deux CP mais davantage en CP1 (étapes de la structure narrative au nombre de $5-6-7 / 7$ pour $70 \%$ des élèves de $\mathrm{CPp} 1$ contre $50 \%$ des élèves de CP2 à l'issue de la période 2);

- une augmentation de l'utilisation des organisateurs textuels dans les deux CP mais de façon plus significative en CP1 (à relier avec l'évolution de la structure narrative). Par ailleurs les organisateurs textuels et connecteurs sont plus variés en $\mathrm{CP} 1$ comme le montre la présence de temporels mais aussi d'argumentatifs et de présentatifs ( $45 \%$ des élèves de CP1 ont recours à ces trois catégories) sont concernés contre $16 \%$ des élèves de $\mathrm{CP} 2$ à l'issue de la période 2 ).

- une augmentation de l'utilisation des procédés anaphoriques dans leur variété et une utilisation plus modérée des anaphores pronominales dans les deux CP. Cependant, le panel des anaphores est plus important en CP1 qu'en CP2. En CP1 les anaphores pronominales occupent des fonctions syntaxiques plus variées qu'en CP2 (sujet et complément en CP1). Par ailleurs, en CP1, on observe une augmentation importante des relations anaphoriques marquées par le déterminant.

- une progression de l'emploi pertinent des temps du récit oral dans les deux classes. On observe qu'en CP1 plus aucun élève n'utilise un seul temps. Le couple Imparfait / Passé Composé est présent dans $40 \%$ des récits oraux en $\mathrm{CP} 1$ et dans $33 \%$ des récits oraux en $\mathrm{CP} 2$. Les temps les plus fréquents en $\mathrm{CP} 1$ sont exclusivement ceux de l'oral : Imparfait / Passé Composé / Présent. En revanche, en CP2, ce sont ceux de l'oral et de l'écrit : Imparfait / Passé Composé / Présent / Passé Simple.

- un abandon de la progression thématique constante unique par tous les élèves des deux CP au profit de progressions plus complexes (au recours exclusif à une progression à thème constant est privilégié le recours à une progression à thème linéaire combinée à une progression à thème éclaté mais aussi à thème ponctuellement constant, etc.).

La proportion d'élèves qui ne proposent pas de récit est quasiment la même dans les deux classes. La classe de CP1 progresse donc sur tous les indicateurs retenus pour les besoins de l'analyse. Il en est de même de la classe de CP2 mais dans des proportions moins importantes. Par ailleurs, les organisateurs textuels, les connecteurs et les procédés anaphoriques sont plus variés en CP1. Il semble donc que, à milieu socioculturel équivalent, le manque de diversité des connecteurs proposés par les élèves du CP2 résulte d'une absence de manipulation des récits répondant à une structure narrative de forme quinaire et non pas à des difficultés linguistiques. En effet, l'enseignante de la classe de CP1 lit des histoires à ses élèves quotidiennement et ces derniers manipulent matériellement et oralement tous les jours des albums de littérature de jeunesse pour participer aux comités de lecture. Rappelons que les productions orales analysées reposent sur un album de jeunesse qui a été lu plusieurs fois par l'enseignante et permettent quotidiennement aux élèves de manipuler et d'écouter des histoires composées d'obstacles, de complications, d'actions, de résolutions.

A l'issue des périodes 1 et 2 , les principaux résultats sont les suivants pour le CP1 : 


\begin{tabular}{|c|c|c|}
\hline \multirow{2}{*}{ Indicateurs } & \multicolumn{2}{|c|}{ CP1 } \\
\hline & Période 1 & Période 2 \\
\hline $\begin{array}{l}\text { Organisateurs } \\
\text { textuels et } \\
\text { connecteurs }\end{array}$ & $\begin{array}{l}61.05 \% \text { des élèves utilisent des } \\
\text { organisateurs textuels pour introduire } \\
\text { plus de la moitié des étapes de la } \\
\text { structure narrative } \\
-100 \% \text { utilisent des organisateurs } \\
\text { textuels et des connecteurs temporels } \\
-22.2 \% \text { utilisent des organisateurs } \\
\text { textuels et des connecteurs } \\
\text { argumentatifs } \\
-5.5 \% \text { utilisent des organisateurs } \\
\text { textuels et des connecteurs spatiaux }\end{array}$ & $\begin{array}{l}\text { Evolution significative } \\
\text { 100\% des élèves } \\
\text { organisateurs textuels et des } \\
\text { connecteurs temporels } \\
\text { textuels et des connecteurs } \\
\text { argumentatifs } \\
\rightarrow 50 \% \text { utilisent des organisateurs } \\
\text { textuels et des connecteurs spatiaux }\end{array}$ \\
\hline $\begin{array}{l}\text { Les étapes de } \\
\text { la structure } \\
\text { narrative }\end{array}$ & $\begin{array}{l}50 \% \text { des élèves utilisent plus de la } \\
\text { moitié des étapes de la structure } \\
\text { narrative attendue pour satisfaire à la } \\
\text { restitution du récit de départ }\end{array}$ & $\begin{array}{l}\text { 88.8\% des élèves } \\
\text { Evolution significative }\end{array}$ \\
\hline $\begin{array}{l}\text { La cohésion } \\
\text { énonciative }\end{array}$ & $\begin{array}{l}22.25 \% \text { des élèves utilisent les temps de } \\
\text { l'énonciation de type discours (imparfait } \\
\text { / passé composé, présent) et } 22.25 \% \text { des } \\
\text { élèves utilisent ces mêmes temps } \\
\text { combinés avec les temps de } \\
\text { l'énonciation de type historique } \\
\text { (imparfait / passé simple) } \\
55.5 \% \text { des élèves utilisent un seul temps }\end{array}$ & $\begin{array}{l}\text { 83.25\% des élèves utilisent les } \\
\text { temps de l'énonciation de type } \\
\text { discours et } 16.65 \% \text { utilisent les } \\
\text { deux types d'énonciation } \\
\text { simultanément } \\
\text { Evolution significative dans la } \\
\text { maîtrise par la classe de la cohésion } \\
\text { énonciative puisque plus aucun } \\
\text { élève n'utilise un seul temps }\end{array}$ \\
\hline $\begin{array}{l}\text { Les procédés } \\
\text { anaphoriques }\end{array}$ & $\begin{array}{l}38.85 \% \text { des élèves utilisent des procédés } \\
\text { anaphoriques variés } \\
\text { ( } 61.5 \% \text { des élèves utilisent des procédés } \\
\text { anaphoriques réduits à la substitution } \\
\text { pronominale) }\end{array}$ & $\begin{array}{l}88.8 \% \text { des élèves } \\
\text { Evolution significative }\end{array}$ \\
\hline $\begin{array}{l}\mathrm{La} \\
\text { progression } \\
\text { thématique }\end{array}$ & $\begin{array}{l}83.25 \% \text { des élèves utilisent une } \\
\text { progression thématique complexe }\end{array}$ & $\begin{array}{l}\text { 100\% des élèves } \\
\text { Evolution significative }\end{array}$ \\
\hline
\end{tabular}

En fin de période 2, nous observons que tous les élèves ont progressé significativement quant à la maîtrise des outils linguistiques assurant la cohésion textuelle de leur récit. Ainsi les organisateurs textuels, les connecteurs et la progression thématique complexe sont convoqués par tous les élèves. De même, près de 9 élèves sur 10, ont recourt aux principales étapes de la structure narrative et à des procédés anaphoriques variés, et plus de 8 élèves sur 10 ont recourt à une cohésion énonciative de type historique. 
Notre étude confirme celle de Fayol, Gombert et Mouchon (1991) et la prolonge aussi. Il apparaît en effet que, si le support est important, la manipulation quotidienne de cet exercice l'est tout autant. Enfin, plus largement, nous soulignons que le registre discursif de l'enseignante, que nous avons décrit extrêmement interactif, faisant une place significative à la parole des élèves et reposant sur une exploitation du support littéraire, semble avoir une incidence directe sur les performances de ses mêmes élèves. Enfin, souvent convoquée (Prêteur et Rouquette, 1992), la variable socioculturelle semble ne pas être déterminante puisque nous avons pu établir une différence quantitative mais aussi et surtout qualitative dans le recours aux principaux indicateurs linguistiques du récit narratif oral entre deux classes de CP présentant des profils socioculturels identiques mais prises en charge par des enseignantes ayant des registres discursifs différenciés.

\subsection{Performances culturelles}

Proposé un mois après la rentrée, le premier questionnaire est composé de 16 items se répartissant de la façon suivante : 9 relatifs aux correspondants québécois, 1 sur les activités scolaires liées à la correspondance, 4 sur la ville de Marseille et enfin 2 sur la lecture familiale et ses représentations. Proposé à six mois d'intervalle en avril, le second questionnaire est sensiblement le même que le premier, à la différence que les deux dernières questions concernent la communication du projet de correspondance dans la famille de l'élève.

Nous analyserons successivement la nomination et le choix du correspondant, les connaissances encyclopédiques relatifs aux deux espaces géographiques convoqués, la prise de conscience des activités scolaires liées au projet et leur verbalisation dans l'espace privé.

\subsubsection{Nomination et choix du correspondant}

Le questionnaire interroge d'abord l'élève sur le nom de son correspondant et les raisons de son choix. $\mathrm{Si}$, à l'initiale du projet épistolaire, $45 \%$ des élèves sont capables de nommer leur correspondant et de justifier leur choix, $55 \%$ ne se souviennent pas du nom de leur correspondant. Un enfant qui ne peut nommer son correspondant est néanmoins capable de préciser une des raisons de son choix. L'absence de nomination n'est donc pas à interpréter comme du désintérêt.

M. obtient la palme du nombre de raisons avec 5 critères de choix (pizza, rose, son ami, le cerf-volant, le volant).T. et $P$. expriment 4 raisons. Les 5 autres élèves expriment un ou deux critères de choix. La couleur aimée revient à 5 reprises dans les critères de choix et les animaux 6 fois. 3 élèves ont choisi un autre « soi-même » : « elle aime tout ce que j'aime ». La fonction identitaire du même est prégnante à ce stade initial. Par exemple, L. dit qu'il a choisi son correspondant pour la marque de sa console de jeu, sa couleur préférée et conclut « tout pareil que moi ». On remarquera qu'un élève est conscient de ne pas avoir été l'auteur du choix en disant que c'est l'enseignante qui a choisi pour lui.

Six mois plus tard, on passe de 45 à 80 , voire $100 \%$ de capacité à nommer son correspondant : 12 sur 15 le nomment directement et les 3 autres avec aide (lecture du nom la photo qui est reconnue parmi un large panel). Les raisons évoluent sensiblement. Deux élèves font assumer le choix à leur enseignante. T. qui avait exprimé 4 raisons en octobre reprend celle du feutre et ajoute le fait que ce soit un garçon.

Les raisons restent cohérentes et s'approfondissent. Pour les filles, la beauté intervient 4 fois. Bastien dit de son correspondant : «il m'adore ». Il semble que la fonction identitaire du même a évolué vers une relative décentration et une prise en compte de l'autre et de ses goûts.

\subsubsection{Connaissances encyclopédiques}

\subsubsection{Savoirs déclaratifs au début du projet}

Pour faire un premier point sur les connaissances encyclopédiques liminaires, on distinguera celles qui concernent les correspondants et celles qui concernent le contexte géographique local. 
A l'initiale du projet, tous les élèves savent que leur correspondant vit très loin de chez eux mais $55 \%$ n'ont pas mémorisé le prénom de leur correspondant. $45 \%$ des élèves connaissent le nom du pays, soit le Canada, $40 \%$ ne peuvent pas répondre à cette question, $10 \%$ répondent Québec ${ }^{14}$ et $5 \%$ proposent le nom de l'école de leur correspondant, soit Val-Joli.

Les deux tiers des élèves ne connaissent pas le nom de la ville des correspondants, $5 \%$ la connaissent ${ }^{15}$, $10 \%$ disent Canada, 10\% le nom de l'école et 5\% Marseille. Ces confusions compréhensibles dénotent une curiosité réelle à propos de l'espace géographique de leur correspondant puisque les deux tiers des élèves souhaitent lui poser des questions. Seulement $10 \%$ des élèves parlent du château Frontenac, emblème de la ville de Québec dont la photo a été envoyée par les correspondants.

Quant aux connaissances locales, les résultats montrent une ignorance toute relative chez de très jeunes élèves au seuil de l'école élémentaire. Si 58\% des élèves ne connaissent pas le nom de leur quartier, la même proportion connaît celui de leur ville, soit 58,5\% Marseille, 10,5\% France, 10,5\% Consolat (nom du quartier), 5,25\% « Marseille-Consolat », réponse qui agrège les noms de la ville et du quartier et enfin $5,25 \%$, l'OM (club de football marseillais).

A la question sur le nom de leur pays, 39\% ne répondent pas à la question, 28\% Marseille, 16,5\% le nom de leur quartier, $5,5 \%$ Turquie et 5,5\% l'OM.

Il est normal que les connaissances encyclopédiques liées à la culture locale soient plus développées au niveau micro qu'à celui macro, c'est-à-dire que des élèves de 6 ans soient plus familiers de la nomination de leur quartier que de celle de leur ville ou encore de leur pays que seulement $5 \%$ peuvent nommer. $A$ contrario, $45 \%$ des enfants nomment le pays de leur correspondant mais seulement $5 \%$ le nom de leur ville. Les connaissances liminaires concernant la culture géographique de l'autre sont plus importantes au niveau macro qu'au niveau micro alors que c'est l'inverse au niveau des connaissances culturelles locales où le sujet arrive moins à se décentrer.

Sur la base de ces premiers constats, on peut se demander si l'impact du projet épistolaire culturel se mesure non seulement au niveau de l'empan des connaissances mais aussi au niveau de la décentration et de l'ouverture à l'autre et au général, ce qui engage le rapport à la culture.

\subsubsection{2. Évolution des connaissances encyclopédiques}

10 élèves sur 15 évoluent notablement après six mois d'implication dans le projet. La distinction pays, ville, école commence à se mettre en place même si ce n'est pas encore le cas de tous les élèves qui ont besoin, par exemple, d'une aide avec la prononciation de la première syllabe de la réponse attendue. $\mathrm{Si}$, en octobre, quelques élèves nommaient le nom de l'école de leur correspondant car ils venaient de recevoir une photo électronique commentée en classe, il n'y a plus qu'un élève qui le cite spontanément. Par contre, ils arrivent à lire de manière autonome le nom sur la photo. Tous les élèves ont un grand plaisir à localiser le Canada sur la carte du monde.

A propos de la question nouvelle dans le second questionnaire, "que sais-tu sur le Canada? », les réponses proposées sont les suivantes : «Grand pays avec des choses pas ici/ grand pays / pays froid, chiens de traîneau, neige/carnaval fête/ loin + bateau + avion + océan)». Les connaissances dont l'évolution est notable sont liées aux photos et cartes envoyés par les correspondants et commentés collectivement en classe et non à des savoirs communiqués par le professeur.

Paradoxalement, l'impact des envois des correspondants est plus fort que celui des activités sur la ville de Marseille qui ont fait l'objet d'activités pas toujours reliées explicitement à la correspondance. Il y a parfois un déficit de sens pour un élève de 6 ans à qui son professeur demande de commenter une photo de Notre-Dame-de la Garde ou du Prado. Ceci peut expliquer que l'évolution des connaissances encyclopédiques de l'espace géographique lointain du correspondant soit plus importante que celles de son espace géographique proche. 
L'hypothèse selon laquelle le fait de travailler sur la culture de sa ville permettrait de s'approprier plus facilement des connaissances sur une culture étrangère reste valide mais elle mérite d'être nuancée ou infléchie. Il est probable que la prise de conscience de son identité géographique rend possible la découverte de celle de l'autre avec qui l'élève entre en contact épistolaire. Et parfois, en fonction des dispositifs didactiques et de leurs tenants et aboutissants, c'est-à-dire du sens qu'ils revêtent pour l'élève, certaines connaissances sont plus ou moins appropriées. En l'occurrence, les connaissances sur Québec sont plus importantes que celles sur Marseille car les élèves marseillais ont reçu plus de cartes et d'envois de leurs correspondants québécois qu'ils ne leur en ont faits.

\subsubsection{Rapport à la culture, à sa culture et à celle de l'autre}

On s'appuiera sur les réponses concernant la représentation du mode de vie des correspondants québécois et sur les questions que les élèves marseillais souhaitent leur poser car ces deux entrées permettent de prendre en compte la curiosité et l'ouverture à l'autre.

$\mathrm{Si}$, en octobre, $45 \%$ des élèves n'ont aucune représentation et $55 \%$ considèrent que les Québécois sont « différents » et " gentils ». Six mois plus tard, il n'y a plus qu'une seule absence de réponse, tous les élèves de $\mathrm{CP}$ se sont forgé une représentation et ils essaient de répondre à la question en se référant à leur propre mode de vie et en formulant un jugement de valeur (voir en annexe un panel de réponses).

Les questions proposées aux correspondants sont révélatrices de la curiosité des élèves envers leurs correspondants, de la nature de leur ouverture qui peut dire aussi en creux leurs attentes par rapport au projet interculturel. En six mois, on passe de $70 \%$ à $15 \%$ des élèves qui n'ont pas de question à poser. La curiosité s'est accentuée avec le développement de la correspondance et la réception des envois des correspondants. Les questions posées sont essentiellement des indices de goût identitaires des élèves. Par exemple, Y. demande à son correspondant s'il sait jouer au football, au rugby et au tennis et précise qu'il a marqué beaucoup de buts tout en ajoutant les noms des joueurs de football qu'il aime.

\subsubsection{Prise de conscience des activités scolaires liées au projet et verbalisation dans l'espace privé.}

Tous les élèves sauf un sont capables de parler des activités liées au projet de correspondance. La réponse développée de $\mathrm{A}$. est révélatrice et exemplaire de la prise de conscience par les élèves des activités menées dans le cadre du projet culturel et de la correspondance. Voici quelques exemples :

- «j'ai fabriqué une carte d'identité/ pour comme ça on l'envoie par le courrier/ comme ça ils nous l'envoient et nous + si on lui envoie pas + notre courrier il va être déçu et on va il va être fâché et nous on est obligé de lui fabriquer un courrier »

- « elle nous a expliqué la maîtresse on met des des feuilles et une carte $+\mathrm{hm}$ pour fabriquer/ comme on écrit notre nom de famille notre prénom + et $\mathrm{hm}$ comme on met aussi si on est une fille ou un garçon + on fait une croix sur une hm carré tout petit ++ comme ça on:: on barre on leur dit qu'on est une fille et après on regarde la date de naissance/ on est né le combien + on l'écrit et après on signe on signe on écrit notre prénom et après on fait comme des gragrabouillages »

- « nous allons on va préparer toutes les cartes d'identité et vous allez l'envoyer à nos correspondants »

La description des activités scolaires liées au début du projet de correspondance, un mois après son lancement, est déjà précise. L'investissement des élèves est fort et découle probablement de celui de leur enseignante.

En mars, tous les élèves parlent du projet et évoquent les activités passées, présentes et même à venir ou projetées. T. qui avait répondu «sais pas » à l'ensemble du questionnaire en octobre répond : «de l'écriture, on raconte sur les dinosaures, un herbier c'est un livre sur les plantes et aussi on va faire un 
livre sur Marseille. » En octobre, les activités mentionnées étaient celles présentes et il n'y avait pas encore de projection dans le futur avec des objectifs contractualisés. Ainsi, A. qui est la seule à mentionner les napperons promis par les Québécois, précise :

- « ils vont nous envoyer des ronds comme des nappes avec des étiquettes »

- « fais des dessins + des cœurs + des animaux + une maison avec de l'herbe »

- « je vais dire chers correspondants et je vais envoyer un dessin sur la ville de Marseille + sur les chiens et les animaux »

Nous observons une nouvelle décentration due à l'impact d'une dynamique de projet qui a duré six mois. Les élèves sont dans la relation, le dialogue et ont des projets d'envoi (25\% parlent nommément d'envoi) et citent les noms des supports travaillés pour les correspondants (carte, prospectus).

La question sur l'évocation du projet dans l'espace privé n'est posée qu'en avril ${ }^{16}$. Elle repose sur le postulat de l'importance pour l'enfant de parler de l'école à la maison pour qu'il fasse des liens entre les différents apprentissages et donne du sens à ceux-ci.

$35 \%$ n'en ont pas parlé à leurs parents mais $65 \%$ ont parlé du projet à leur famille dont deux précisent même qu'ils écrivent à leur correspondant avec l'aide de leur parent. Même si c'est un garçon qui a ramené le plus de choses pour son correspondant et qu'il en a parlé à sa maman, sa petite et sa grande sœur, les trois quarts des élèves qui ont verbalisé dans leur famille le projet scolaire sont des filles. Il y a aussi $20 \%$ qui en parlent alors qu'ils ne bénéficient pas de soutien en lecture ni de lectures offertes dans leur environnement familial. Deux élèves mentionnent un oubli mais une envie d'en parler. Bastien ajoute : «je vais le dire en sortant»

Malgré les difficultés inhérentes à tout projet sollicitant l'investissement de plusieurs acteurs, le dispositif de correspondance internationale scolaire se révèle positif pour les élèves qui se sont donc ouverts sur l'autre tout en construisant une conscience identitaire de leurs (dé)goûts et une première culture de leur ville. Au total, ce projet s'est révélé être constructif : il a favorisé la construction d'une communauté interactionnelle de lecteurs et d'auteurs générant des apprentissages dont témoignent les performances analysées tant au plan linguistique que culturel. Il a permis notamment de favoriser un rapport positif à la langue et à la culture qui sont complètement imbriqués (en donnant du sens à la pratique épistolaire) mais il a permis aussi de donner plus de sens à la pratique de la correspondance en en devenant le contenu d'enseignement et en évoluant à travers l'appréhension de la culture de « soi » et de la culture de l' « autre » conformément au phénomène décrit par Reuter (2007).

\section{Références bibliographiques}

Applebee, A.-N. (1975). Thechikf's concept of story : Agetwo to seventeen, Chicago, The University of Chicago Press.

Bakhtine, M.-M. (1929). Le marxisme et la philosophie du langage : essai d'application de la méthode sociologique en linguistique. Paris : éd. de Minuit, trad. Marina Yaguello (1977)

Barré de Miniac, C. (2002). Le rapport à l'écriture. Une notion à plusieurs dimensions. Pratiques, 113-114, 29-40.

Bautier, E et Rochex, J.Y (1998). L'expérience scolaire des nouveaux lycéens. Paris : Armand Colin.

Brown, P. et Levinson, S. (1978). Universals in language use : Politeness phenomena. In E.Goody (Ed.) Questions and politeness. Strategies in social interaction. Cambridge : CUP, 56-289.

Brown, P. et Levinson, S. (1987). Politeness. Some universals in language use. Cambridge : CUP.

Bucheton, D. dir. (1997). Conduites d'écriture au collège et au lycée professionnel. Versailles : CRDP.

Bucheton, D. et Chabanne, J.C. dir. (2002). Parler et écrire pour penser, apprendre et se construire. L'écrit et l'oral réflexifs. Paris : PUF, collection Éducation et formation.

Burgos, M. et Leenhardt, J. (1991). La raison et passion européenne. Le français aujourd'hui, 95. 
Bourdieu, P. (1992. Les règles de l'art. Genèse et structure du champ littéraire. Paris : Seuil.

Charolles, M. (1978). Introduction aux problèmes de la cohérence textuelle. In Charolles M. et Peytard J. (Eds.). «Enseignement du récit et cohérence du texte ». Langue française, 38.

Charolles, M. (1976). Grammaire de texte. Théorie du discours. Pratiques, 11-12, 133-154.

Chartier, A.M., Clesse, C. et Hébrard, J. (1998). Produire des textes au cycle 2. Paris : Hatier.

Crinon, J. (2002). Écrire le journal de ses apprentissages dans Bucheton, D et Chabanne, J.C. (Dir.) Parler et écrire pour penser, apprendre et se construire. L'écrit et l'oral réflexifs. Paris : PUF, collection Éducation et formation.

Daunay, B. (2007), Séminaire THEODILE sur l'évaluation des performances comme objet de recherche, Université Charles-de- Gaulle, Lille III.

Delcambre, I. et Reuter, Y. (Dir.). (2002). Images du scripteur et rapports à 1'écriture, Pratiques, 113-114, 7-28.

Dolz, J., Noverraz, M. et Schneuwly, B. (Dir.). (2001). S'exprimer en français. Séquences didactiques pour l'oral et pour l'écrit. Bruxelles : DeBoeck et Larcier.

Dolz, J. et Pasquier, A. (1993). Argumenter... pour convaincre: initiation aux textes argumentatifs. Cahier du Service de Français, 31. Genève : DIP.

Dumortier, J.L. (2001). Lire le récit de fiction. Pour étayer un apprentissage : de la théorie à la fiction. Bruxelles : De Boeck Duculot.

Fayol M. (1992). L'écrit : Perspectives cognitives. In Entretiens Nathan. Vol. 2. Paris : Nathan

Fayol, M. (1981). L'organisation du récit écrit ; son évolution chez l'enfant de 6 à 10 ans. Thèse pour le Doctorat d'Etat, Université de Bordeaux II (3 vol.).

Fayol, M., Gombert, J.-E. et Mouchon, S. (1991). «L'emploi de quelques connecteurs dans les récits », Repères, 3, $87-98$.

Genette, G. (1997). L'euvre d'art. La relation esthétique. Paris : Seuil.

Grize J.-B. (1996). Logique naturelle et communication. Paris : PUF

Goffman, E. (1974). Les rites d'interaction, Paris, Minuit.

Goffman, E. (1971). La mise en scène de la vie quotidienne, t1 La présentation de soi, t2 Les relations en public, trad. française 1973, Paris, Editions de Minuit.

Kerbrat-Orecchioni, C. (1996). La conversation, Seuil, collection Mémo.

Kerbrat-Orecchioni C. (1992). Les interactions verbales t. II, Paris, A. Colin

Lahire, B. (1993). Culture écrite et inégalités scolaires. Sociologie de "l'échec scolaire » à l'école primaire. Lyon: Presses universitaires de Lyon.

Lebrun, M. (2005). Posture critique et geste anthologique. Faire vivre la littérature à l'école. Cortil-Wodom : EME, collection Proximités didactiques.

Lebrun, M. et Romain, C. (2010a). Echange culturel et évolution du rapport à la culture chez des élèves de 6 ans », Actes du colloque international «Problématiques culturelles dans l'enseignement-apprentissage des languescultures, mondialisation et individualisation : approche interdisciplinaire, Université de Tallinn, Estonie, 2008, Synergie Pays riverains de la Baltique.

Lebrun, M. et Romain, C. (2010b). L'impact d'une correspondance scolaire sur la construction identitaires d'élèves défavorisés de 6 ans, Colloque International La construction identitaire à l'école - Approches pluridisciplinaires, Université Montpellier 2 - I.U.F.M. de Montpellier, France, 2009, TREMA.

Miller, P.-J. et Sperry, L.-L. (1988). Early talk about the past : The origins of conversational stories of personnal experience. Journal of Child Language, 15, 293-315.

Petit, M. (2002). L'éloge de la lecture. La construction de soi. Paris : Belin. 
Prêteur, Y. et Rouquette, L. (1992). « L'incidence d'une pédagogie fonctionnelle autour du livre de jeunesse sur les compétences orales et écrites d'enfants de grande section de maternelle de milieux sociaux contrastés », Revue française de pédagogie, 98, 29-40.

Reuter, Y. (1996). Enseigner et apprendre à écrire. Paris : ESF.

Reuter, Y. (Ed.). (2007). Dictionnaire des concepts fondamentaux des didactiques. Bruxelles : Édition de Boeck université.

Reuter, Y. (Dir.), Equipe Théodile, (2007). Une école Freinet. Fonctionnements et effets d'une pédagogie alternative en milieu populaire, Paris : L'Harmattan.

Reuter, Y. (2005). Statut et usages de la notion de genre en didactique (s) : quelques propositions dans Journée ceditec/ théodile, Le genre comme outil, comme objet, comme enjeu, 9 déc. 2005.

Romain, C. (2007). L'emploi des temps et des organisateurs textuels narratif d'élèves de 9 ans à 14 ans issus de milieux socioculturels contrastés, Revue des sciences de l'éducation, Entreprise commune des facultés, départements, instituts et écoles universitaires francophones d'éducation du Canada, XXXIII, 1, 209-235.

Schneuwly, B. (1988). Le langage écrit chez l'enfant. Neuchâtel : Delachaux et Niestlé

Schneuwly, B. et Dolz, J. (1997). Les genres scolaires. Des pratiques scolaires aux objets d'enseignement. Repères, $15,27-40$.

Tauveron, C. et alii. (2005). Vers une écriture littéraire. Paris : Hatier.

Umiker-Sebeok, D.-J. (1979). Preschool children's intraconversational narratives. Journal of Child Language, 6, 91109.

Von, R. (1999). Linguistique et communication verbale. In M. Gilly, J-P. Roux et A. Trognon (éds). Apprendre dans l'interaction. Analyse des médiations sémiotiques. Presses Universitaires de Nancy et de l'Université de Provence.

Vion, R. (1992). La communication verbale. Analyse des interactions. Paris : Hachette Université.

Waters, H.-S. et Hou, F.-T. (1987). Children's production and recall of narrative passages. Journal of Experimental Child Psychology, 44, 348-363.

\footnotetext{
${ }^{1}$ Elèves de 6 ans scolarisés à l'école élémentaire Saint Louis Consolat, Marseille, France.

${ }^{2}$ Un échange culturel consiste à faire prendre conscience aux élèves des éléments caractéristiques de leur propre culture tout en les faisant pénétrer la culture des autres. Il permet ainsi une évolution de leurs représentations.

${ }^{3}$ Correspondance scolaire avec des élèves canadiens de 7ans scolarisés à l'école Val Jolie, Québec, Canada.

${ }^{4}$ L'établissement scolaire est inscrit dans une zone d'éducation prioritaire / ambition réussite / zone violence et s'inscrit dans les quartiers réputés « défavorisés » de la ville.
}

${ }^{5}$ Cf. Lebrun M. et Romain C., 2010ab

${ }^{6}$ L'établissement scolaire est inscrit dans une zone d'éducation prioritaire / ambition réussite / zone violence et s’inscrit dans les quartiers réputés « défavorisés » de la ville.

${ }^{7}$ Chaque jour, une demi-heure est consacrée à la découverte et à la lecture d'un album. Ce moment est préparé avec attention (installation de chacun, climat de confiance et de calme, règles de prises de parole...) Quotidiennement, un temps de lecture personnelle d'environ 15 minutes est proposé aux enfants, l'enseignante lit aussi. En fin de semaine, une séance d'environ 45 minutes est organisée pour procéder au vote et organiser l'affichage collectif. Pour l'enseignante, il est essentiel que ces moments quotidiens se déroulent dans un climat de confiance mutuelle.

8 « Nous appelons donc image(s) du scripteur (ou du lecteur) la construction d'une configuration qui articule, au sein de déclarations et/ou de pratiques et/ou de textes, des attitudes, des valeurs, des conceptions, des savoirs sur l'écriture, la lecture, les textes, les savoirs et leurs relations. Ou, pour le dire plus (trop) simplement, la construction de la /des façon(s) dont le scripteur se représente les objets et les 
compétences mentionnées et se situe par rapport à eux au travers de ses discours, de ses activités et de ses textes. » (Delcambre et Reuter, 2002, 19)

${ }^{9}$ Chaque enfant peut choisir un texte quelconque (dans l'album ribambelle, dans un livre de la bibliothèque ou bien de la maison). Chaque candidat prépare sa lecture où il veut (à la maison, en classe), quand il veut et aussi longtemps qu'il le désire. Une séance spéciale de lecture libre à haute voix est organisée deux à trois fois par mois. Ils s'inscrivent en début de semaine. 7 candidats peuvent se succéder au cours de la séance qui dure environ 30 à 40 minutes.

${ }^{10}$ Chaque jour, une demi-heure est consacrée à la découverte et à la lecture d'un album. Ce moment est préparé avec attention (installation de chacun, climat de confiance et de calme, règles de prises de parole...) Chaque jour également, un temps de lecture personnelle d'environ 15 minutes est proposé aux enfants, l'enseignante y participe. Chaque vendredi, une séance d'environ 45 minutes est organisée pour procéder au vote et organiser l'affichage collectif. Il est essentiel pour l'enseignante que ces moments quotidiens se déroulent dans un climat de confiance mutuelle.

${ }^{11}$ Transcription intégrale des récits oraux qui ont donné lieu à une analyse prenant en compte les étapes de la structure narrative utilisée par l'élève, l'emploi des temps, les procédés anaphoriques, la progression thématique et les organisateurs textuels ainsi que les connecteurs.

12 Banque d'outils à l'évaluation diagnostique de l'éducation nationale, Fiche d'évaluation EGCADB01 : Le petit chaperon rouge, Grande Section et Cours Préparatoire.

${ }^{13}$ G. Solotareff, 2001, Loulou, Ecole des Loisirs.

${ }^{14}$ On peut considérer que la nomination de Québec soit une bonne réponse puisque Québec est à la fois une ville et une province.

${ }^{15}$ Seulement la moitié de ceux qui avaient donné comme réponse Québec à la question de nomination du pays reprennent Québec à la question de nomination de la ville.

${ }^{16}$ En octobre, la question porte sur les lectures offertes à la maison et la représentation de l'intérêt d'apprendre à lire. 